\title{
P-1137 Effect of Fasting Ramadan on Different laboratory parameters in Egyptian patients with type 2 Diabetes Mellitus Maram M.Aboromia ${ }^{1}$, Hanan M.Amer ${ }^{1}$, Yara M.Eid ${ }^{1}$, Yomna kabany ${ }^{2}$ ${ }^{1}$ Ain-Shams University Hospitals - Internal Medicine department, Cairo -Egypt ${ }^{2}$ National Diabetes Institute, Cairo-Egypt.
}

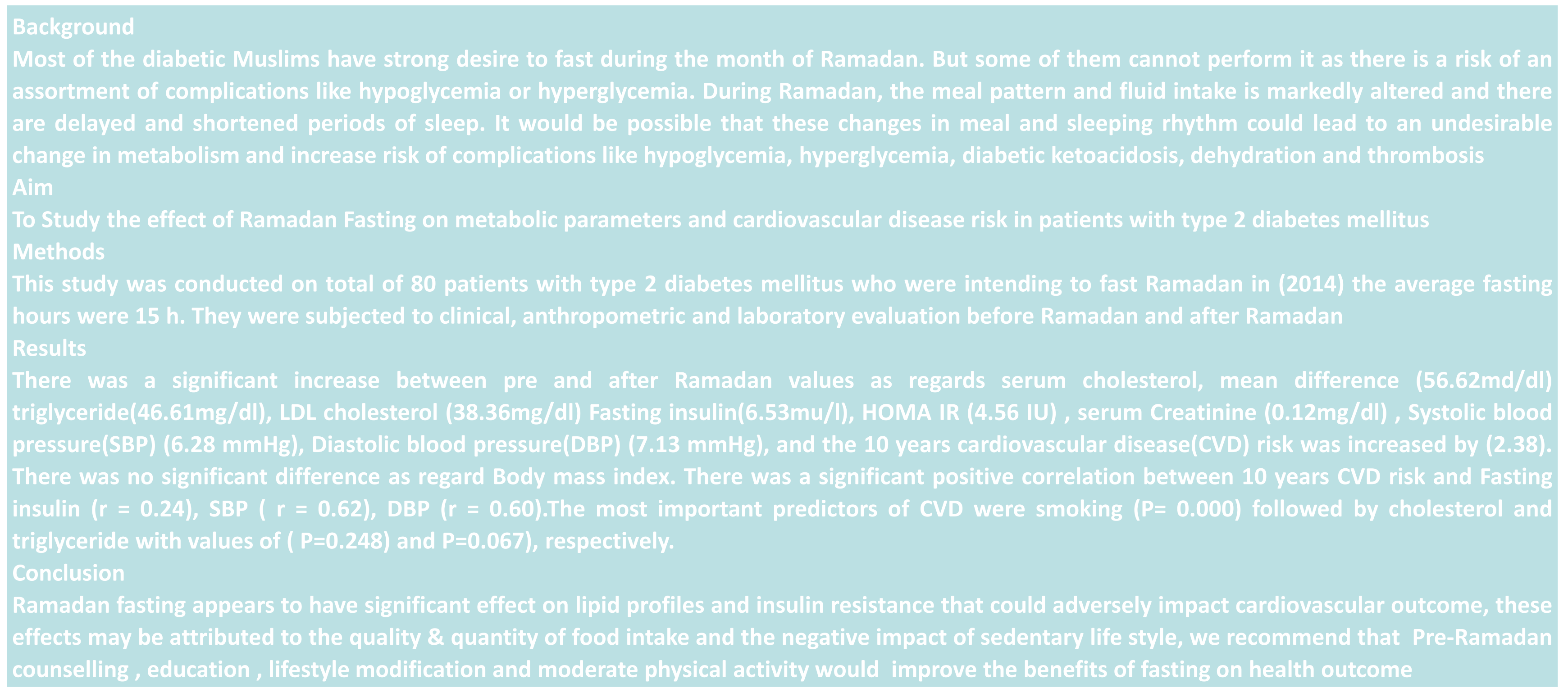

\section{Introduction}

Diabetes burden is expected to continuously rise in MENA region and other areas in the globe (1), yet the burden of this predominantly Muslim populated areas has a special particularity in Ramadan fasting. While exempted from fasting many Muslim patients choose to fast (2). The existing literature on the effects of Ramadan fasting on cardiovascular outcomes and related factors are controversial (2), in addition studies addressing this point in diabetic populations are rather few.

Aim of the study : To study the impact of Ramadan fasting on metabolic parameters, cardiovascular risk and anthropometry in Egypt Study Design:

A cohort study that was conducted upon 80 people with type 2 diabetes who were intending to fast Ramadan (2014) with an average of 15 hours of fasting , the study was approved by the local faculty ethical committee. Patients were invited for full clinical and anthropometric examination and laboratory evaluation one day prior to Ramadan and after Feast by one day.

Laboratory evaluation included Fasting blood sugar, 2hr Post Prandial blood sugar , HbA1c\%,Fasting serum lipid profile (total cholesterol, LDL cholesterol, HDL cholesterol and triglycerides),Creatinine, albumin/ Creatinine ratio, Highly sensitive c reactive protein (HSCRP) and fasting insulin for calculation of HOMA-IR .Also Framingham risk Score was applied to detect cardiovascular risk score pre Ramadan and after Ramadan.

Patients with type 1 diabetes, chronic kidney disease > stage 3 , hepatic disease CHILD B and C classification and pregnant patients were excluded from the study and provided with appropriate medical advice .

\section{Results}

The mean age of the study population was $49.91 \pm 10$ with a gender distribution of $19(23,75 \%)$ males and $61(76.25 \%)$ females.

Smokers constituted $22.5 \%$ of the study population while $67.5 \%$ of the patients were hypertensive.

1. "IDF Diabetes Atlas - 7th Edition." IDF Diabetes Atlas - Home, IDF, 2015, www.diabetesatlas.org/.

2. Mazidi M, Rezaie P, Chaudhri O, et al. The effect of Ramadan fasting on cardiometabolic risk factors and anthropometrics parameters: A systematic review. Pakistan Journal of Medical Sciences. 2015;31(5):1250-1255. doi:10.12669/pjms.315.7649.
Table 1 showing Comparison of the study variables (paired t-test)

\begin{tabular}{|c|c|c|c|c|c|}
\hline Variable & $\begin{array}{l}\text { Before Ramadan } \\
\text { mean } \pm \text { SD }\end{array}$ & $\begin{array}{l}\text { After Ramadan } \\
\text { mean } \pm \text { SD }\end{array}$ & MD & $\mathrm{t}$ & $P$ value \\
\hline $\mathrm{FBS} \mathrm{mg} / \mathrm{dl}$ & $164.2 \pm 43.1$ & $212.5 \pm 190.5$ & $0.787-$ & -0.23 & 0.8 \\
\hline PPBS mg/dl & $281.4 \pm 83.9$ & $287.3 \pm 85.7$ & -5.9 & -0.88 & 0.38 \\
\hline HbA1c\% & $8.1 \pm 2.02$ & $8 \pm 2.13$ & 0.06 & 0.554 & 0.6 \\
\hline $\mathrm{TC} \mathrm{mg} / \mathrm{dl}$ & $145.5 \pm 46$ & $202.13 \pm 39.9$ & -56.62 & -10.7 & $<0.001$ \\
\hline TG $\mathrm{mg} / \mathrm{dl}$ & $150.03 \pm 84.2$ & $196.6 \pm 133.2$ & -46.6 & -2.92 & 0.004 \\
\hline HDL-c mg/dl & $42 \pm 15$ & $46 \pm 6.9$ & -3.9 & -2.5 & 0.014 \\
\hline LDL-c mg/dl & $78.5 \pm 42.1$ & $117.6 \pm 46.07$ & -39.03 & -7.17 & $<0.001$ \\
\hline Fasting insulin U/L & $6.1 \pm 3.7$ & $12.6 \pm 8.2$ & -6.537 & 2.3 & $<0.001$ \\
\hline HOMA-IR & $2.42 \pm 1.6$ & $7.02 \pm 8.9$ & -4.56 & -4.5 & $<0.001$ \\
\hline $\mathrm{BMI} \mathrm{kg} / \mathrm{m}^{2}$ & $36.1 \pm 5.9$ & $36 \pm 5.6$ & 0.08 & 0.5 & 0.62 \\
\hline Waist hip ratio & $0.906 \pm 0.11$ & $0.907 \pm 0.11$ & -0.0005 & -0.45 & 0.65 \\
\hline Serum creatinine mg/dl & $0.81 \pm 0.2$ & $0.94 \pm 0.3$ & -0.12 & -5.1 & $<0.001$ \\
\hline Albumin/creatinine ratio & $21.2 \pm 6.5$ & $21.4 \pm 6.2$ & -0.04 & -1.84 & 0.07 \\
\hline SBP mmHg & $134.9 \pm 10.8$ & $141.2 \pm 11.9$ & -6.28 & -14.32 & $<0.001$ \\
\hline DBP mmHg & $94.7 \pm 10.7$ & $101.8 \pm 11.3$ & -7.13 & -9.97 & $<0.001$ \\
\hline 10 years CVD risk & $5.18 \pm 7.8$ & $7.6 \pm 9.9$ & -2.38 & -4.09 & $<0.001$ \\
\hline
\end{tabular}

Correlation of 10 year CVD risk score and studied variables showed a significant positive correlation with systolic blood pressure, diastolic blood pressure and fasting insulin $(\mathrm{r}=0.62 ; 0.6$ and 0.24 respectively).Also binary logistic regression analysis for predictors of 10 years CVD score after Ramadan fasting showed that the most important predictors of CVD were Smoking followed by Cholesterol and Triglyceride.

Lipid profile, Fasting insulin , HOMA-IR , SBP, DBP, HsCRP and serum creatinine significantly increased after Ramadan fasting while glycemic parameters showed a non-significant rise (Table 1)

\section{Conclusion}

We concluded that Ramadan fasting in people with type 2 diabetes appears to have significant effect on lipid profiles and blood pressure that could adversely impact cardiovascular risk.

Current guidance is more focused on glycemic control and oral hypoglycemic agents and insulin dose adjusments. Nonetheless Cardiovascular disease risk assessment and guidance prior to Ramadan fasting should not be overlooked.

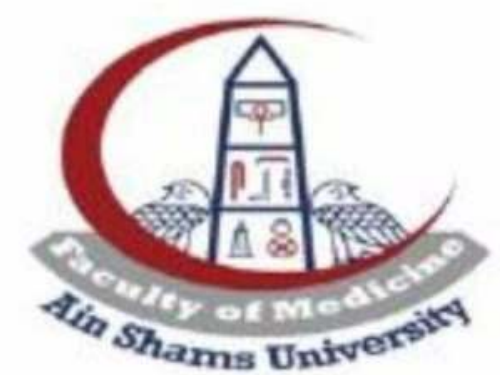

